\title{
Management of an incidental finding of right internal jugular vein agenesis
}

\author{
Vincent KF Kong *, FANZCA, FHKAM (Anaesthesiology), CJ Jian, MB, BS, R Ji, MS, MB, BS, \\ Michael G Irwin, MD, FHKAM (Anaesthesiology)
}

Department of Anaesthesia, HKU-Shenzhen Hospital, 1 Haiyuan Road, Futian District, Shenzhen, China

*Corresponding author: vincentkong@hku.hk

Hong Kong Med J 2017;23:414-5

DOI: 10.12809/hkmj166086

\section{Case report}

A 43-year-old woman was referred to the University of Hong Kong-Shenzhen Hospital in August 2016 with a 10-year history of hepatolithiasis. Computed tomography (CT) of the abdomen demonstrated multiple stones in the right posterior portion of the liver, the common biliary duct, and the gallbladder. Dilatation and inflammation of both intra- and extra-hepatic ducts were apparent and an elective right hepatectomy along with a Roux-en-Y hepaticojejunostomy was arranged. Preoperative physical examination was normal apart from hypertension $(168 / 105 \mathrm{~mm} \mathrm{Hg})$. Laboratory tests revealed a microcytic, hypochromic anaemia (haemoglobin, $97 \mathrm{~g} / \mathrm{L}$ ), hyperuricaemia, and mildly elevated alkaline phosphatase. Liver/renal function, clotting profile, chest X-ray, and electrocardiogram were all normal. General anaesthesia was induced intravenously with propofol and remifentanil using a target-controlled infusion (Marsh model) under the guidance of the Bispectral Index monitoring system (Covidien, Boulder [CO], US). Tracheal intubation was performed following administration of rocuronium and anaesthesia maintained intravenously with intermittent positive pressure ventilation in oxygen and air. The patient was positioned for right internal jugular vein (IJV) cannulation.

Pre-insertion sonographic evaluation of the right cervical region (SonoSite $\mathrm{M}$-Turbo, Bothell [WA], US) using a linear, high-frequency transducer (HFL38, 6-13 MHz) revealed only a single pulsatile vessel that was non-compressible and suggestive of the right carotid artery. The characteristic pulsatile blood flow was confirmed by Doppler. There was no evidence of the right IJV despite repositioning of the patient's head, use of minimal pressure on the probe with colour flow mapping, and the application of Valsalva manoeuvre. Ultrasonography of the left side showed normal anatomy with good size of IJV. Following a brief discussion, the consultant anaesthetist and the surgeon decided to proceed with surgery without a central venous catheter. At the end of liver resection, the patient began to develop hypotension that was marginally responsive to fluid resuscitation and moderate-dose phenylephrine infusion through the large-bore peripheral lines. The operation lasted approximately 5 hours with a total blood loss of $350 \mathrm{~mL}$. Tracheal intubation was continued postoperatively and the patient was transferred to the intensive care unit (ICU). Central venous cannulation was not attempted by ICU physicians and the patient was extubated on postoperative day 1 and discharged from the ICU on postoperative day 4 . The patient was followed up by the attending and consultant anaesthetists after surgery. The incidental finding of her neck condition was explained and she agreed to undergo further investigations for a possible vascular anomaly.

Ultrasonography (iU Elite model with a L12-5 transducer; Philips Medical System, Bothell [WA], US) of the neck by a radiologist on postoperative day 5 confirmed the absence of thrombosis and the right IJV. The left IJV was normal (diameter, 15-19 mm). Multisection CT angiography of the head and neck region revealed agenesis of the right IJV (Fig). There was no other vascular anomaly in the head and neck region. The patient was discharged from hospital on day 11 postoperatively.

\section{Discussion}

Non-visualisation of the right IJV on two-dimensional sonographic scanning can be due to operator (eg suboptimal patient positioning, inappropriate machine setting, and excessive pressure on the probe) and patient (eg vascular thrombosis, congenital anomalies such as hypoplasia, agenesis) factors. Congenital agenesis of the IJV is an extremely rare anomaly. ${ }^{1}$ The risks associated with central venous cannulation of the left IJV or the right subclavian vein in our patient before surgery may have outweighed the benefits. The IJVs are the principal vessels for cerebral venous drainage. Injury and/or thrombosis of the left IJV can still occur even if proper precautions are taken during catheterisation. Disruption of the alternative channels of venous drainage from the cranial cavity in a patient with congenital agenesis of the IJV may have serious consequences. Vascular malformations in the head and neck region result from embryological developmental deformities and 


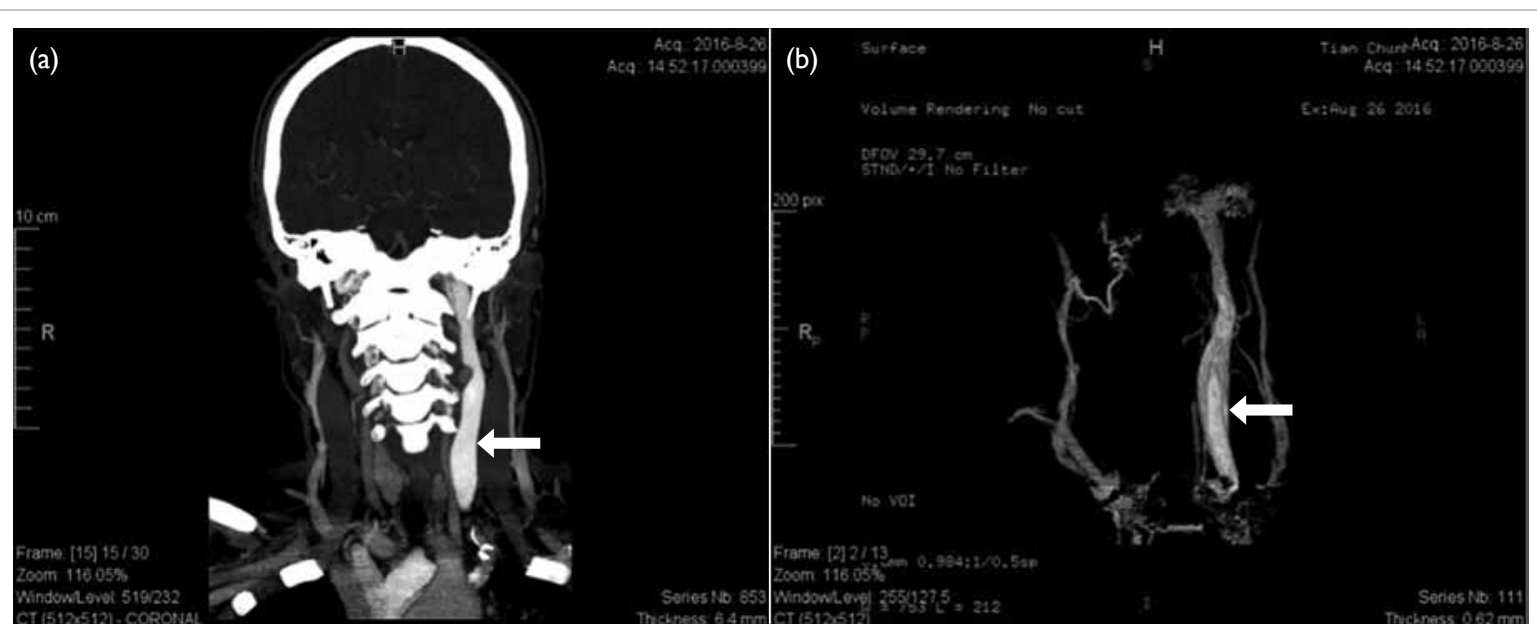

FIG. Multisection computed tomographic angiography (CTA) of the head and neck region showing congenital agenesis of the right internal jugular vein (IJV); the normal left IJV is seen (arrows)

(a) CTA and (b) bone-subtraction CTA

can co-exist asymptomatically. Further diagnostic analysis before cannulation of her right subclavian vein would have provided extra safety.

Low central venous pressure (CVP) during anaesthesia reduces surgical blood loss in major hepatic resection, ${ }^{2}$ and central venous cannulation of the right IJV to enable CVP monitoring has become a routine practice in many places prior to major hepatectomy. Less invasive techniques such as peripheral venous pressure and external jugular venous pressure measurement allow an acceptable estimation of the CVP with less associated morbidity and mortality. Stroke volume variation (SVV) derived from the Vigileo-FloTrac system (Edwards Lifesciences, Irvine [CA], US) can be a safe and effective alternative to conventional CVP monitoring during hepatic resection. ${ }^{3}$ The FloTrac system is based on arterial pulse contour analysis and does not require external calibration, thermodilution, or dye dilution. Unlike CVP that is a favoured but static measure of intravascular volume, SVV monitors dynamically the physiological interactions of the heart and lungs in mechanically ventilated patients to not only estimate fluid status but also predict fluid responsiveness. A high SVV of $10 \%$ to $20 \%$ is associated with significantly less blood loss during liver resection. ${ }^{4}$ Nonetheless a multiparametric approach should be adopted to guide fluid management in complicated cases because every haemodynamic variable has limitations and interferes with other variables.

Anaesthetists should function as perioperative physicians to minimise patient harm and create extra value to the episode of patient care. A simple, focused ultrasound examination of the neck during preoperative assessment can diagnose variation in vessel position or abnormalities of the vessel. It has been recommended by the National Institute for Health and Care Excellence in the United Kingdom since $2002^{5}$ and this report further supports its routine use. Early recognition of these anomalies enables extra precautions to be taken (eg discussion with the patient and surgeons for alternative anaesthetic plans, cannulation sites, monitoring strategies, and further investigation before surgery) to reduce patient harm.

\section{References}

1. Kayiran O, Calli C, Emre A, Soy FK. Congenital agenesis of the internal jugular vein: an extremely rare anomaly. Case Rep Surg 2015;2015:637067.

2. Wang WD, Liang LJ, Huang XQ, Yin XY. Low central venous pressure reduces blood loss in hepatectomy. World J Gastroenterol 2006;12:935-9.

3. Reineke R, Meroni R, Votta C, et al. Enhanced recovery after open hepatectomy with minimally invasive haemodynamic monitoring: A successful challenge. A comparative study from a single institution. Clin Nutr ESPEN 2016;12:e53-4.

4. Dunki-Jacobs EM, Philips P, Scoggins CR, McMasters KM, Martin RC 2nd. Stroke volume variation in hepatic resection: a replacement for standard central venous pressure monitoring. Ann Surg Oncol 2014;21:473-8.

5. Kong V, Yuen M, Irwin M. Perioperative ultrasonography: Ultrasound and vascular cannulation. CPD Anaesthesia 2007;9:3-9. 